\title{
Controlling biology by radio
}

Radio waves and iron nanoparticles could hold the key to targeted perturbation of cells deep inside living tissue.

Light is an indispensible medium for observation and is increasingly used for manipulating living systems. But its tendency to interact with molecules - the property that makes it so useful-is a nuisance when the target is deep inside tissue. In comparison, radio waves readily pass through living tissue but can rapidly heat iron nanoparticles, which can then transfer heat to a temperature-sensitive cation channel (TRPV1) and trigger calcium ion $\left(\mathrm{Ca}^{2+}\right)$ influx in neurons (Huang et al., 2010).

Friedman and colleagues now adapt this system to regulate gene expression in vivo (Stanley et al., 2012). They used retrovirus to deliver a histidine (His)-tagged TRPV1 channel gene and an engineered insulin gene into cells and then injected the cells into nude mice, where they formed tumors. The authors injected 20-nm iron oxide nanoparticles coated with anti-His antibody into the tumors. Exposing the mice to radio waves heated the particles and triggered $\mathrm{Ca}^{2+}$ influx and activation of the insulin gene, resulting in an $\sim 50 \%$ increase in plasma insulin. Because the promoter controlling the insulin gene responds only to sustained increases in intracellular $\mathrm{Ca}^{2+}$, the particles did not affect insulin levels in the absence of radio waves.

Compared to optical approaches that rely solely on genetically encoded constituents, the requirement for delivering nanoparticles obviates many of the method's advantages. In an effort to overcome this drawback, Friedman and colleagues expressed an engineered ferritin protein in cultured cells so the cells could synthesize their own intracellular iron nanoparticles alongside TRPV1 and the engineered insulin. Exposure to radio waves stimulated insulin gene expression in these cells about two-thirds as efficiently as the exogenous-particle approach.

Although less well developed, the second approach is of more interest for in vivo applications and could be useful for deep neuronal stimulation via $\mathrm{Ca}^{2+}$ influx using just TRPV1 and ferritin. But there is a challenge. TRPV 1 is 839 amino acids in size, and the approach requires coexpression of ferritin. Compared to the $<600$ amino acids for a single ChR2-YFP fusion used in optogenetics, this could present a challenge for both viral delivery and transgenic animal generation. But solutions that allow widespread use of the method will surely come.

\section{Daniel Evanko}

\section{RESEARCH PAPERS}

Huang, H. et al. Remote control of ion channels and neurons through magnetic-field heating of nanoparticles. Nat. Nanotechnol. 5, 602-606 (2010).

Stanley, S.A. et al. Radio-wave heating of iron oxide nanoparticles can regulate plasma glucose in mice. Science 336, 604-608 (2012). 